\title{
HIV Infection as Risk Factor for Death among Hospitalized Persons with Candidemia, South Africa, 2012-2017
}

Nelesh P. Govender, Jim Todd, Jeremy Nel, Mervyn Mer, Alan Karstaedt, Cheryl Cohen, for GERMS-SA ${ }^{1}$

\section{Medscape ACTIVITY}

In support of improving patient care, this activity has been planned and implemented by Medscape, LLC and Emerging Infectious Diseases. Medscape, LLC is jointly accredited by the Accreditation Council for Continuing Medical Education (ACCME), the Accreditation Council for Pharmacy Education (ACPE), and the American Nurses Credentialing Center (ANCC), to provide continuing education for the healthcare team.

Medscape, LLC designates this Journal-based CME activity for a maximum of 1.00 AMA PRA Category 1 Credit(s) ${ }^{\mathrm{TM}}$. Physicians should claim only the credit commensurate with the extent of their participation in the activity.

Successful completion of this CME activity, which includes participation in the evaluation component, enables the participant to earn up to $1.0 \mathrm{MOC}$ points in the American Board of Internal Medicine's (ABIM) Maintenance of Certification (MOC) program. Participants will earn MOC points equivalent to the amount of $C M E$ credits claimed for the activity. It is the CME activity provider's responsibility to submit participant completion information to ACCME for the purpose of granting ABIM MOC credit.

All other clinicians completing this activity will be issued a certificate of participation. To participate in this journal CME activity: (1) review the learning objectives and author disclosures; (2) study the education content; (3) take the post-test with a $75 \%$ minimum passing score and complete the evaluation at http://www.medscape.org/journal/eid; and (4) view/print certificate. For CME questions, see page XXX.

Release date: May 19, 2021; Expiration date: May 19, 2022

\section{Learning Objectives}

Upon completion of this activity, participants will be able to:

Describe the effect of HIV infection and other factors on mortality risk from candidemia, according to an analysis of data from a 6-year sentinel surveillance study in South Africa

Determine the association of intensive care unit admission with mortality risk among adults with HIV infection and candidemia, according to an analysis of data from a 6-year sentinel surveillance study in South Africa

Identify clinical implications of the effect of HIV infection on mortality risk from candidemia, according to an analysis of data from a 6-year sentinel surveillance study in South Africa

\section{CME Editor}

Jill Russell, BA, Copyeditor, Emerging Infectious Diseases. Disclosure: Jill Russell, BA, has disclosed no relevant financial relationships. CME Author

Laurie Barclay, MD, freelance writer and reviewer, Medscape, LLC. Disclosure: Laurie Barclay, MD, has disclosed no relevant financial relationships.

\section{Authors}

Disclosures: Nelesh P. Govender, MBBCh, MMed, MSc Epi, MSc Mycol; Jim Todd, BA, MSc; and Alan Karstaedt, MBBCh, have disclosed no relevant financial relationships. Jeremy Nel, MBChB, has disclosed the following relevant financial relationships: served as an advisor or consultant for Cipla Inc.; Johnson \& Johnson Pharmaceuticals Research \& Development, L.L.C.; Mylan Laboratories Inc.; served as a speaker or a member of a speakers bureau for AbbVielnc. Mervyn Mer, MD, PhD, has disclosed the following relevant financial relationships: served as a speaker or a member of a speakers bureau for Pfizer Inc.; Sanofi Aventis. Cheryl Cohen, MD, PhD, has disclosed the following relevant financial relationships: received grants for clinical research from Sanofi Pasteur.

Author affiliations: University of Cape Town, Cape Town, South Africa (N.P. Govender); University of the Witwatersrand, Johannesburg (N.P. Govender, J. Nel, M. Mer, A. Karstaedt, C. Cohen); National Institute for Communicable Diseases, a Division of the National Health Laboratory Service, Johannesburg, South
Africa (N.P. Govender, C. Cohen); London School of Hygiene and Tropical Medicine, London, UK (J. Todd)

DOI: https://doi.org/10.3201/eid2706.210128

${ }^{1}$ Members of GERMS-SA are listed at the end of this article. 
We determined the effect of HIV infection on deaths among persons $\geq 18$ months of age with culture-confirmed candidemia at 29 sentinel hospitals in South Africa during 2012-2017. Of 1,040 case-patients with documented HIV status and in-hospital survival data, $426(41 \%)$ were HIV-seropositive. The in-hospital casefatality rate was $54 \%$ (228/426) for HIV-seropositive participants and $37 \%(230 / 614)$ for HIV-seronegative participants (crude odds ratio [OR] 1.92, 95\% Cl 1.50$2.47 ; p<0.001)$. After adjusting for relevant confounders $(\mathrm{n}=907)$, mortality rates were $1.89(95 \% \mathrm{Cl} 1.38-2.60)$ times higher among HIV-seropositive participants than HIV-seronegative participants $(p<0.001)$. Compared with HIV-seronegative persons, the stratum-specific adjusted mortality OR was higher among HIV-seropositive persons not managed in intensive care units (OR 2.27, $95 \% \mathrm{Cl} 1.47-3.52 ; \mathrm{p}<0.001)$ than among persons who were (OR 1.56, 95\% Cl 1.00-2.43; $p=0.05$ ). Outcomes among HIV-seropositive persons with candidemia might be improved with intensive care.

C andida is a common cause of healthcare-associated bloodstream infections in South Africa; the estimated national incidence risk in 2016-2017 was 84 (95\% CI 81-86) cases per 100,000 hospital admissions (1). This rate is $\approx 10$-fold higher than that reported in the United States (2). The death rate among patients with Candida bloodstream infections is high and associated with such factors as confirmed or presumed gastrointestinal source of infection, lack of source control, shorter time to positivity of blood cultures, inappropriate or delayed empiric antifungal treatment, lack of consultation with an infectious disease physician, severe sepsis or septic shock, and severity of underlying conditions (3-5). The crude mortality rate associated with candidemia was $43 \%$ in South Africa in 2016-2017 (1). In 2 small case series of HIVseropositive patients with candidemia (Spain, $\mathrm{n}=$ 37 ; Italy, $\mathrm{n}=38$ ), the crude mortality rate was $\approx 60 \%$ $(6,7)$. HIV infection may be a risk factor for death among persons with culture-confirmed candidemia. In a 2014 population-based surveillance study in Spain, 16 (2\%) of 752 patients with candidemia were HIV-seropositive (8). Although HIV infection was not associated with death on univariable analysis (OR 0.51, 95\% CI 0.07-3.93), the study was underpowered. In contrast, in a United States cohort study of 446 adults with candidemia, 22 (5\%) were HIV-seropositive, and HIV infection was associated with a 2-fold increased adjusted hazard of 30-day mortality (hazard ratio 2.02, 95\% CI 1.11-3.72) (9). This effect of HIV infection on death rates may be mediated by factors related to the host (e.g., neutropenia or neutrophil dysfunction in persons with advanced HIV disease) or the fungal pathogen (infection caused by Candida species other than C. albicans or by $>1$ Candida spp.) $(6,7,10-12)$. Such an association has not been described in a high HIV prevalence setting. By using data from a 6-year sentinel surveillance study at hospitals in South Africa, we examined the effect of HIV infection on the risk for 30-day mortality among persons with candidemia.

\section{Materials and Methods}

\section{Study Population}

We included persons $\geq 18$ months of age with an episode of culture-confirmed candidemia identified at 29 sentinel hospitals in South Africa during January 1, 2012-December 31, 2017. The HIV prevalence among inpatients is high at these large urban tertiaryacademic or regional acute-care hospitals (e.g., 60\% at a Cape Town district hospital in 2012-2013) (13). A case was defined as illness in a person in whom Candida spp. was cultured from blood at a laboratory providing diagnostic pathology services to a sentinel hospital. An episode was defined as a 30-day period from the date of the first positive Candida culture. Any positive blood cultures after this period defined a recurrent episode and thus a new case. We excluded children $<18$ months of age because we did not have HIV PCR results to confirm an HIV diagnosis for a sufficient number of persons.

\section{Surveillance Methods}

Cases were reported from public-sector and privatesector pathology laboratories. In general, blood cultures were collected if patients had clinical features of sepsis, including tachycardia, tachypnea, increased or subnormal temperature, a change in sensorium, hypotension, or prostration (14). Viable Candida isolates were submitted to a reference laboratory for specieslevel identification and antifungal susceptibility testing, as previously described (1). Trained nurses or pharmacists identified culture-confirmed cases in the laboratory and interviewed prospectively enrolled participants or their next of kin or reviewed medical charts or electronic laboratory records. Data were collected through a standardized case report form. The main explanatory variable was the participant's HIV infection status, which was determined during the admission for candidemia. HIV status was selfreported during participant interviews or abstracted from the inpatient chart, a child's outpatient immunization card, or laboratory records. An in-hospital outcome with a date of outcome was determined at the end of admission to the acute-care hospital or if 
the participant was transferred to a step-down facility; this outcome was determined at the end of that admission. Outcome was recorded as alive at 30 days for prolonged admissions of $>30$ days after first positive blood culture. Underlying or immediate cause of death was not recorded. Surveillance officers recorded whether a person had been admitted to the intensive care unit (ICU) at any time during hospitalization, but the specific reason for and dates of ICU admission or discharge were not captured. We calculated a quick Pitt score, an abbreviated version of the Pitt bacteremia score, as the sum of individual scores for body temperature of $<35^{\circ} \mathrm{C}$ ( 1 point), systolic blood pressure of $<90 \mathrm{~mm} \mathrm{Hg}$ (1 point), cardiac arrest (1 point), mechanical ventilation (1 point), and altered mental status (1 point) on the day of candidemia diagnosis $(15,16)$.

\section{Data Analysis}

We used participant identifiers to remove duplicate records. We described the characteristics of the study participants by using descriptive statistics. To determine risk factors for death, we used classical Mantel-Haenszel methods to calculate crude case fatality ratio, odds ratio (OR), and $95 \% \mathrm{CI}$ for HIV status and each of the potential confounders (Appendix, https://wwwnc.cdc.gov/EID/article/27/5/210128-App1.pdf). We did not assume that individual participant-level outcomes were statistically independent in this sentinel surveillance study. We therefore used a random-effects logistic regression analysis to explicitly model between-cluster variation at sentinel sites and simultaneously adjust for participant-level confounders (17) (Appendix). We treated the followup period as a fixed 30-day period. We also explored the dose-response effect of HIV on death rates. For this analysis, participants' HIV infection status was recoded as an ordinal variable: HIV-seronegative, HIV-seropositive without advanced immunosuppression (CD4 count $\geq 200$ cells $/ \mu \mathrm{L}$ ), and HIV-seropositive with advanced immunosuppression (CD4 count $<200$ cells $/ \mu \mathrm{L}$ ). Before conducting the analysis, we hypothesized that the effect of HIV infection on death rates would differ among participants with candidemia managed in an ICU and those who were not admitted to an ICU; therefore, we considered ICU admission as an effect modifier a priori. We included participants with recorded dates of positive Candida specimen collection and 30-day outcome in a Kaplan-Meier survival analysis. For 67 participants with an outcome date that coincided exactly with the specimen collection date, we recoded their timeto-outcome to 0.5 days. We explored the association between HIV status and ICU admission by classical and multivariable random effects logistic regression analyses as a post-hoc analysis to explore reasons for the main results.

\section{Ethics}

For GERMS-SA surveillance, annual ethics approvals were sought and obtained from several university ethics committees in South Africa. We also obtained approval from the London School of Hygiene and Tropical Medicine Research Ethics Committee.

\section{Results}

After deduplication, 8,668 cases were detected by GERMS-SA surveillance during the 6-year period. We excluded 3,643 cases diagnosed at non-sentinel sites and 2,462 cases among infants and children $<18$ months of age. Of 2,563 cases diagnosed at sentinel sites, a case report form had been completed for 1,846 $(72 \%)$ (Figure 1). Of those, we retained 1,040 cases with both HIV status and outcome data (56\%). We noted differences in age, sex, year of diagnosis, province in which the diagnosis was made, and Candida species among 717 sentinel-site cases with missing case report forms, 806 cases with completed case report forms but missing HIV status or outcome data, and the 1,040 cases included in the final analysis (Appendix Table 1).

\section{Description of 1,040 Participants Included in the Analysis} Of 1,040 participants, 542 (52\%) were men and boys (Table 1, https://wwwnc.cdc.gov/EID/article/27/6/ 21-0128-T1.htm). The median age of 1,037 participants with recorded age was 37 years (interquartile range [IQR] 23-52 years). Of 1,035 participants with available date of specimen collection, $288(28 \%)$ had a positive Candida blood culture within 72 hours of hospital admission. Overall, 50\% (514/1,023) participants were managed in the ICU during their hospitalization (data on ICU admission were missing for 17 participants). At the time of diagnosis, most $(545 / 1,011,54 \%)$ had a central venous catheter (CVC) in situ, and $24 \%(245 / 1,004)$ were receiving total parenteral nutrition. Of 1,001 participants for whom data was available, $163(16 \%)$ received previous antifungal treatment. A quick Pitt score was calculated for 652/1,040 participants: 319 $(49 \%)$ had a 0 score, $184(28 \%)$ had a 1 score, $126(19 \%)$ had a 2 score, $20(3 \%)$ had a 3 score, and $3(1 \%)$ had a 4 score (Appendix). Thus, 149/652 (23\%) had a quick Pitt score of $\geq 2$. Of 946 case-patients in which Candida species identification was performed at a diagnostic or reference laboratory, 521 (55\%) participants were infected with a Candida species other than C. albicans and 


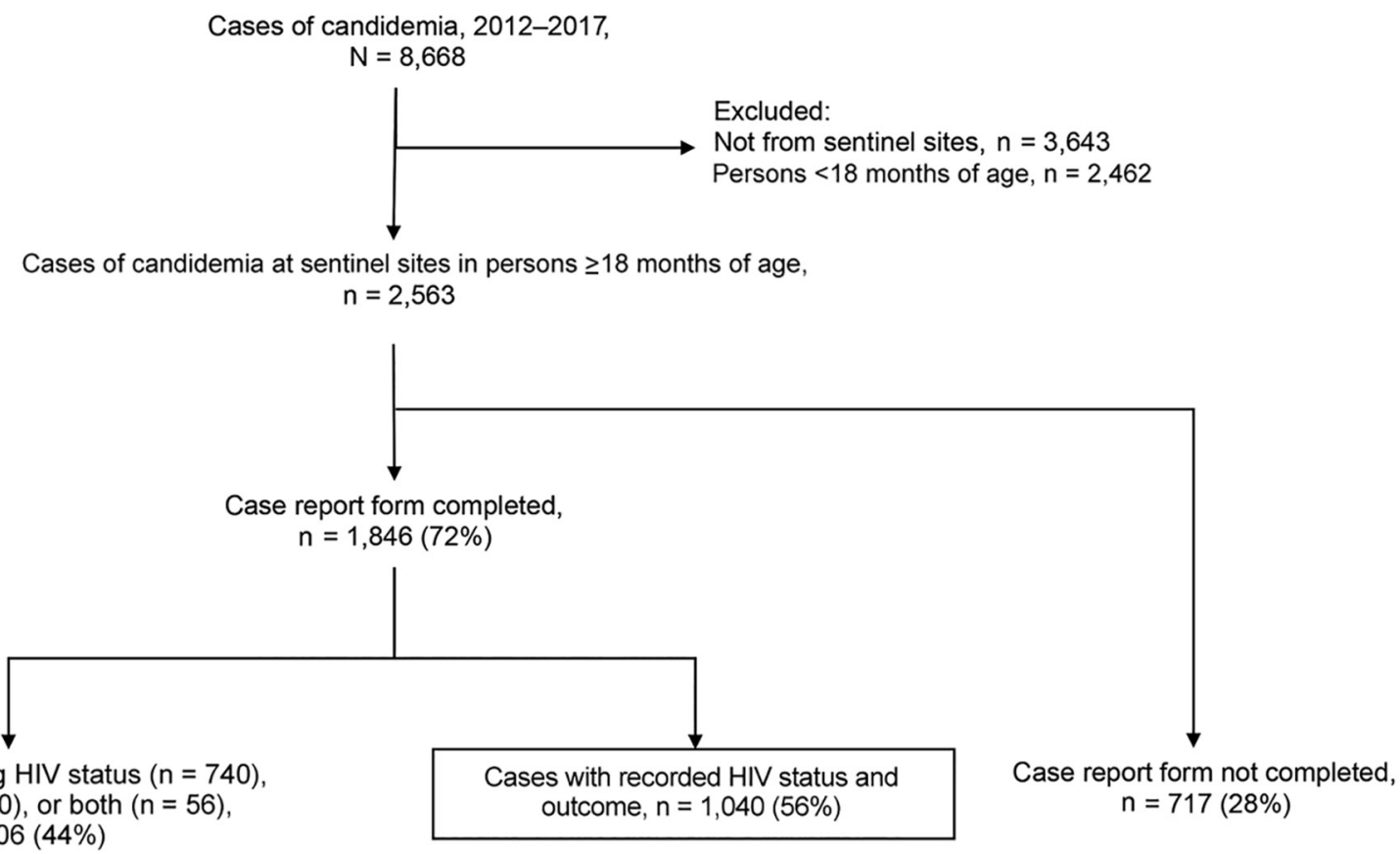

Figure 1. Flowchart demonstrating selection of 1,040 cases of candidemia from a 6-year surveillance period for secondary data analysis, South Africa, 2012-2017.

the remainder with C. albicans; 33 (3\%) case-patients had a mixed Candida infection. Of 1,010 cases, 700 $(69 \%)$ received systemic antifungal treatment to treat candidemia (participants might have received $\geq 1$ antifungal agent: fluconazole $[\mathrm{n}=503]$, amphotericin $\mathrm{B}[\mathrm{n}$ $=265]$, echinocandin [ $n=91]$, or voriconazole [ $n=17]$ ). Of 764 cases with a known Candida species, available antifungal susceptibility data, and applicable Clinical and Laboratory Standards Institute breakpoints (18), $154(20 \%)$ were infected with an antifungal-resistant species. Of these 764, antifungal treatment was recorded for 419 , and 9\% (38/419) had received inappropriate antifungal treatment. Of 488 cases with recorded information, 361 (74\%) had their CVC removed after the candidemia diagnosis. In $4 \%$ of patients $(45 / 1,040)$, evidence of complications of candidemia (deep organ involvement) was indicated in medical charts.

\section{HIV Status and Outcome}

Of the 1,040 case-patients, $426(41 \%)$ were HIVseropositive; we noted several differences in those patients compared with those who were HIV-seronegative (Table 1; Appendix). Among $404 \mathrm{HIV}-$ seropositive persons with available data, 301 (75\%) were antiretroviral treatment-experienced. Among 267 participants in whom CD4 count was recorded near the date of the candidemia diagnosis, the median CD4 count was 133 (IQR 42-309) cells/ $\mu \mathrm{L}$; $166 / 267(63 \%)$ had a CD4 count $<200$ cells $/ \mu \mathrm{L}$. An additional 35 without a CD4 count had a recorded World Health Organization (WHO) clinical stage of HIV disease. Of these 35 case-patients, illness was WHO stage 3 or 4 in 33 case-patients. Of 141 whose records indicated a recently recorded viral load, 65 (46\%) had a viral load of $<400$ RNA copies/mL. Of the 426 HIV-seropositive persons, 153 (36\%) had clinical evidence of HIV-associated wasting. The overall case fatality ratio was $458 / 1,040(44 \%)$. The case-fatality ratio among HIV-seronegative cases was $37 \%(230 / 614)$ versus $54 \%(228 / 426)$ for HIVseropositive cases $(\mathrm{p}<0.001)$.

\section{Risk Factors for 30-Day Mortality}

The crude 30-day case fatality ratio among HIV-seropositive participants was 1.92 (95\% CI 1.50-2.47) times higher than among HIV-seronegative participants $(\mathrm{p}<0.001)$ (Table 2, https:/ / wwwnc.cdc.gov/ EID/article/27/6/21-0128-T2.htm). After adjusting for sentinel hospital, age, sex, year of diagnosis, ICU admission, receipt of systemic antifungal treatment, and Candida species, the odds of 30-day mortality were still 1.89 (95\% CI 1.38-2.60) times higher among HIV-seropositive participants than among HIV-seronegative participants (Table 3); evidence was strong against the null hypothesis $(p<0.001)$. We noted relatively little confounding of the association between HIV status and 30-day mortality by any available explanatory variable. In the final 
model, differences between hospitals accounted for $3 \%$ of the variability in deaths (intracluster correlation coefficient $=0.03 ; p=0.003$ ). We found evidence of interaction of HIV status and ICU admission. The stratum-specific mortality OR was larger in the group not managed in an ICU (OR 2.27, 95\% CI 1.473.52; $\mathrm{p}<0.001)$ than those who were admitted to the ICU (OR 1.56, 95\% CI 1.00-2.43; p = 0.05), although the $95 \%$ CIs overlapped (Table 4 ). In a dose-response analysis, the adjusted odds of 30-day mortality was 1.90 times higher among HIV-seropositive persons with a CD4 count $\geq 200$ cells $/ \mu$ L (95\% CI 1.13-3.20; $\mathrm{p}=0.02$ ) and 2.18 times higher among persons with a CD4 count $<200$ cells $/ \mu \mathrm{L}(95 \%$ CI $1.39-3.42 ; \mathrm{p}=$ 0.001 ) compared with 30-day mortality among HIVseronegative persons (Appendix Table 4).

\section{Kaplan-Meier Survival Analysis}

An outcome date was available for 1,023 participants. Overall, $44 \%(452 / 1,023)$ died within 30 days. The Kaplan-Meier survival curves diverged for HIV-seropositive and HIV-seronegative persons within 3 days of candidemia diagnosis and then remained roughly parallel until day 30 (Figure 2). Evidence was strong against the hypothesis that survival experience did not differ by HIV status $(\mathrm{p}<0.001)$.

\section{Association of HIV Status and ICU Admission}

Among 1,023 participants, a lower proportion (175/422, 41\%) of HIV-seropositive persons than HIV-seronegative persons $(339 / 601,56 \%)$ were admitted to the ICU (crude OR 0.55, 95\% CI 0.42-0.71; $\mathrm{p}<0.001)$. After adjustment for sentinel site, age, sex, and quick Pitt score category $(\mathrm{n}=583)$ (Appendix Table 5), HIV-seropositive participants were $60 \%$ less likely to be admitted to the ICU than HIV-seronegative participants (OR 0.40, 95\% CI 0.25-0.64; $\mathrm{p}<0.001$ ). Among HIV-seropositive participants, a similar proportion with and without advanced HIV disease were admitted to the ICU (72/166 [43\%] vs. 39/99 [39\%]; $\mathrm{p}=0.53$ ). A similar proportion of HIV-seropositive participants receiving and not receiving antiretroviral treatment were admitted to ICU (116/299 [39\%] vs. $50 / 101[50 \%] ; \mathrm{p}=0.06)$.

\section{Discussion}

In this surveillance study of hospitalized persons with candidemia in South Africa, the prevalence of HIV infection was $41 \%(95 \%$ CI $38 \%-44 \%)$. The 30 -day mortality rate was almost twice as high among HIV-seropositive persons as among HIV-seronegative persons (OR 1.89, 95\% CI 1.38-2.60; p<0.001), after adjusting for relevant confounders. The effect of HIV infection on death rates was estimated to be stronger among

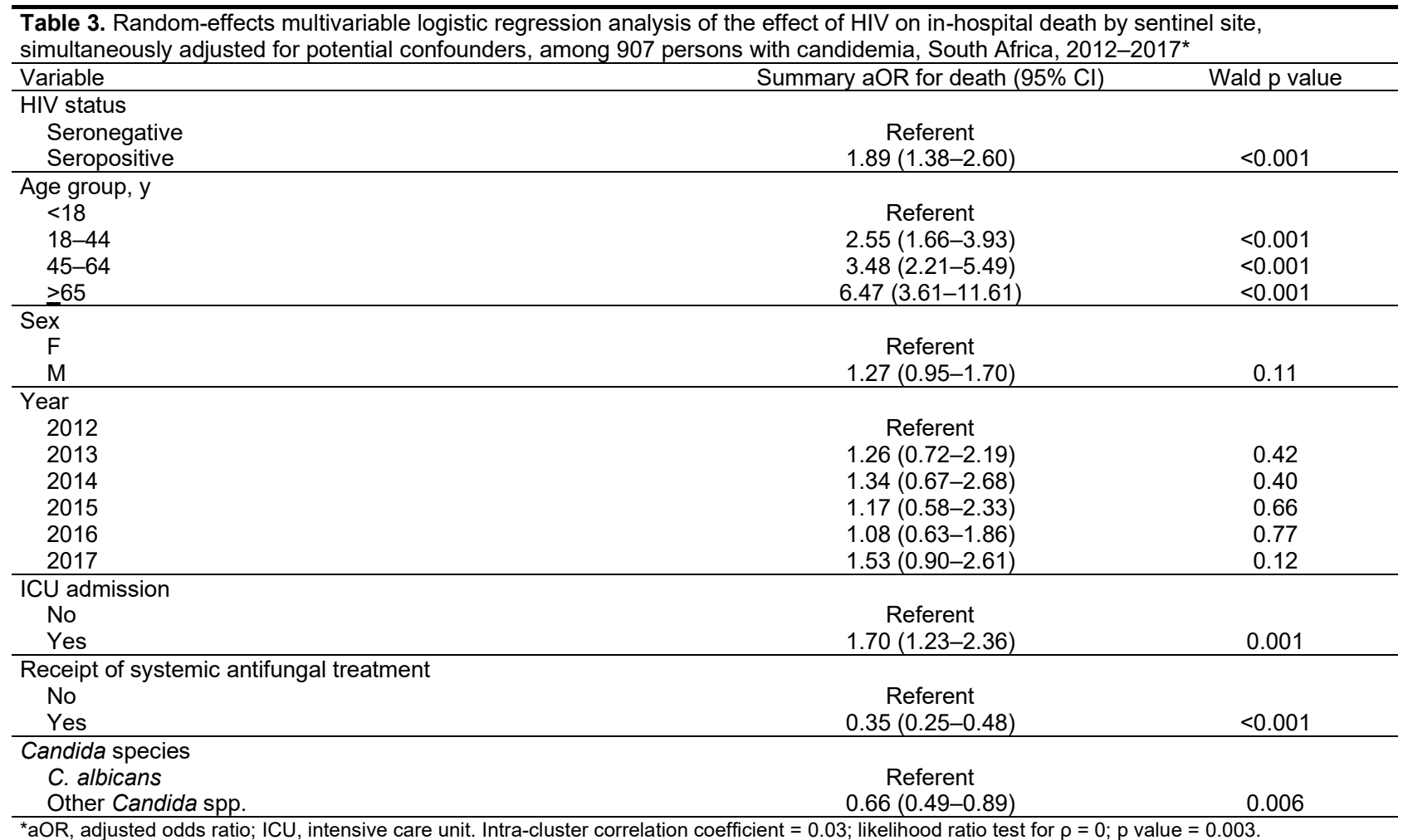




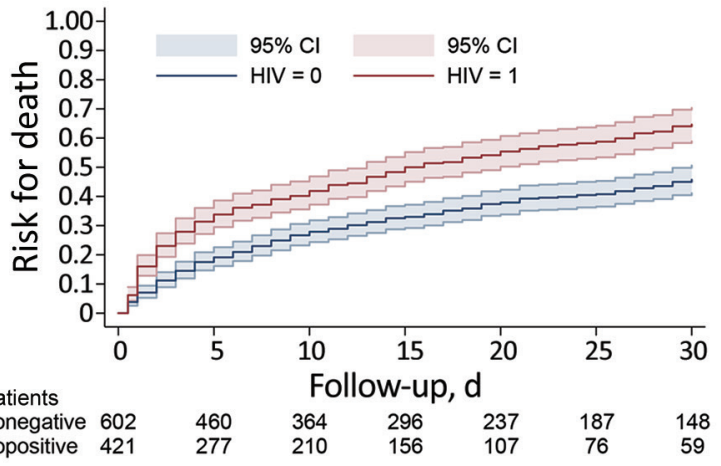

Figure 2. Kaplan-Meier analysis for 1,023 participants with candidemia during a 30-day period after the diagnosis of cultureconfirmed candidemia by HIV infection status (outcome date missing for 17 participants), South Africa, 2012-2017. HIV = 0: HIV-seronegative; HIV = 1: HIV-seropositive; $p$ value for log-rank test $<0.001$.

those not managed in an ICU than those who were, though the $95 \%$ CIs overlapped. HIV-seropositive persons also had a substantially lower risk for ICU treatment during their admission than those who were HIV-seronegative.

The overall crude mortality rate associated with candidemia of $44 \%$ (95\% CI $41 \%-47 \%)$ reported in this study is higher than that reported in resourcerich settings (19), possibly owing to differences in our hospitalized cohort. The overall median age was lower, a large proportion were critically ill, and amphotericin B deoxycholate and azoles were the mainstay of treatment, whereas few patients received echinocandins. In our large study, we found a crude mortality rate among HIV-seropositive patients of $54 \%$, which is comparable to the $60 \%$ mortality rate reported in 2 case series $(6,7)$. Our main results are consistent with a United States cohort study that found that HIV infection was associated with a 2 -fold increased adjusted hazard of 30-day mortality (9). We believe that this association between HIV and death among persons with candidemia is biologically plausible. First, the mechanism for an increased risk for death among HIV-seropositive persons may operate at the individual level. Despite a large proportion having received previous antiretroviral treatment, the median CD4 count among hospitalized HIV-seropositive participants with candidemia in this study was 133 cells $/ \mu \mathrm{L}$, and almost two thirds had advanced HIV disease (defined by the WHO as a CD4 count of $<200$ cells $/ \mu \mathrm{L})(20)$. This finding is consistent with reports of virologic failure and treatment interruption among an increasing proportion of HIV-seropositive patients at acute-care hospitals in South Africa (21). Many persons with advanced HIV disease have neutropenia or neutrophil defects $(10,11)$. This innate immune defect may be a consequence of abnormal progenitor stem cell growth, a deficiency of granulocyte colony-stimulating factor, bone marrow infiltration by opportunistic infectious agents or malignant cells, treatment with particular medicines, or autoimmune phenomena (10). The alteration in the innate immune response may then reduce the clearance of Candida from the bloodstream, despite appropriate antifungal treatment. People with advanced HIV disease might also have been admitted primarily for management of disseminated tuberculosis, cryptococcal disease, or Pneumocystis pneumonia, which are associated with high death rates (20). In addition, more than one third of HIV-seropositive participants in this study were recorded to have evidence of HIV-associated wasting. Patients with a poorer nutritional state or loss of lean body mass have worse outcomes (22).

Second, an increased risk for death may be a consequence of differential treatment practices at the sentinel hospitals for HIV-seronegative and HIV-seropositive persons. We found that HIV-seropositive persons were $60 \%$ less likely to have received intensive care during their hospitalization. Several studies in Italy have documented the large proportion of patients who are managed for candidemia in general medical wards (23-25). In addition, the adjusted effect of HIV infection on 30-day mortality rates was stronger among participants who were not managed in an ICU during their hospital stay. This finding suggests that intensive care and monitoring of patients

Table 4. Interaction between HIV and intensive care unit admission on in-hospital death among 907 persons with candidemia, adjusted for potential confounders, South Africa, 2012-2017*

\begin{tabular}{lcc}
\hline Variable & Stratum-specific aOR for death $(95 \% \mathrm{Cl})$ & Wald test $\mathrm{p}$ value $\dagger$ \\
\hline Admitted to ICU & & \\
HIV-seronegative & Referent & \\
HIV-seropositive & $1.56(1.00-2.43)$ & 0.05 \\
\hline Not admitted to ICU & Referent & \\
HIV-seronegative & $2.27(1.47-3.52)$ & $<0.001$ \\
HIV-seropositive &
\end{tabular}

*aOR, adjusted odds ratio; ICU, intensive care unit.

†Overall likelihood ratio test for interaction $p$ value $=0.22$. Case-fatality ratio for those admitted to an ICU: HIV-seropositive $(94 / 152 ; 62 \%)$, HIV-

seronegative $(157 / 301 ; 52 \%)$ versus those not admitted to an ICU: HIV-seropositive (117/224; 52\%), HIV-seronegative (76/230; 33\%). 
with candidemia, including those who are HIV-seropositive, might reduce mortality rates. However, in a resource-limited setting, the number of ICU beds is restricted. The criteria for admission to a public-sector ICU in South Africa includes an assessment of the severity of the acute or underlying illness and whether organ dysfunction can realistically be reversed. These criteria apply equally to those with and without HIV infection, according to a survey of critical care physicians in South Africa, although no data exist on the proportion of HIV-seropositive persons who were eligible for ICU admission but were not referred or were turned down $(26,27)$. Among HIV-seropositive participants in our study, similar proportions of persons with advanced HIV disease who were receiving antiretroviral treatment were or were not admitted to ICU, suggesting that these factors were not the sole criteria for admission.

We confirmed several well-described risk factors for death among patients with candidemia, including inappropriate or no antifungal treatment, ICU admission, increased age, and a quick Pitt score of $\geq 2$ (28). Removal of a CVC following diagnosis of candidemia was protective against death. A limitation of this study is that it was a secondary data analysis and was not specifically designed or powered to answer whether HIV infection was associated with death among patients with candidemia. However, the study was conducted in a high HIV prevalence setting, our sample size was large, and we found strong and consistent evidence against the null hypothesis after adjustment for confounding. Selection bias was a limitation because we excluded $60 \%$ of cases diagnosed at sentinel hospitals for whom data for the main exposure and outcome variables were missing. We also found differences among those who were included and excluded from the analysis. For instance, we included a larger proportion of cases among adults 18-44 years of age, cases caused by $C$. albicans, and cases from outside Gauteng Province in the analysis (Appendix). Data were also missing for confounder variables, and we excluded several potentially critical confounders from the main multivariable analysis. However, including these variables in a smaller dataset for multivariable analysis did not change the point estimate for the main exposure effect, although the 95\% CI was wider (Appendix Table 3 ). In $\approx 20 \%$ of cases, HIV status was self-reported by participant or next-of-kin interview if a clear record of HIV status was not in the chart. If HIV-seropositive persons underreported their actual infection status, it might have weakened the association with death demonstrated in this study. Because we were unable to adjust for unmeasured factors, such as the presence or severity of diagnosed or undiagnosed underlying conditions, residual confounding is also possible. We conducted this study in an upper middle-income country, and participants were recruited at urban sentinel hospitals with ICU facilities; therefore, the results might not apply to all hospital populations. Cause of death was not recorded, and we were thus unable to estimate the number of deaths directly attributable to candidemia among either HIV-seropositive or HIV-seronegative participants. We excluded children $<18$ months of age from this analysis and cannot comment on whether HIV exposure or infection is associated with death in this population.

A key strength of this study was that it was nested within a large active surveillance system; laboratory audits were conducted to ensure that all culture-confirmed cases were captured. The main outcome measure was in-hospital death, a clear endpoint that was unlikely to have been misclassified. Given that most persons with HIV infection live in sub-Saharan Africa and the risk for healthcare-associated infections, including those caused by antimicrobial-resistant fungi, is becoming increasingly critical in this region $(1,29-$ 31 ), our findings might have broader implications than similar studies and are an essential addition to the literature.

In conclusion, we found that the overall crude mortality rate associated with candidemia was high, and HIV-seropositive persons were at $\approx 2$-fold increased adjusted risk for all-cause in-hospital death, compared with their HIV-seronegative counterparts. This effect on mortality rates was weakened among those admitted to the ICU, though HIV-seropositive persons were substantially less likely to have received intensive care. We recommend a high index of suspicion for candidemia among admitted HIVseropositive persons, regardless of the presence of classical risk factors. We further recommend that HIV-seropositive persons with suspected candidemia rapidly begin appropriate early antifungal treatment, that they be investigated to identify an infection source and control measures instituted, and that they should be considered for intensive care and monitoring to reduce deaths. Where feasible, consultation with an infectious disease specialist would enable this level of care.

Members of GERMS-SA: John Black, Shareef Abrahams, Vanessa Pearce (Eastern Cape Province); Anwar Hoosen, Vicky Kleinhans, Masego Moncho (Free State Province); Alan Karstaedt, Caroline Maluleka, Charl Verwey, Charles Feldman, David Moore, Gary Reubenson, Khine Swe Swe 
Han, Jeannette Wadula, Jeremy Nel, Kathy Lindeque, Maphoshane Nchabeleng, Nazlee Samodien, Nicolette du Plessis, Nontombi Mbelle, Nontuthuko Maningi, Norma Bosman, Ranmini Kularatne, Sharona Seetharam, Teena Thomas, Theunis Avenant, Trusha Nana, Vindana Chibabhai (Gauteng Province); Adhil Maharj, Asmeeta Burra, Fathima Naby, Halima Dawood, Jade Mogamberry, Koleka Mlisana, Lisha Sookan, Praksha Ramjathan, Prasha Mahabeer, Romola Naidoo, Sumayya Haffejee, Yacoob Coovadia Khine Swe Swe Han, Nomonde Dlamini, Surendra Sirkar (KwaZulu-Natal Province); Ken Hamese, Ngoaka Sibiya, Ruth Lekalakala (Limpopo Province); Greta Hoyland, Jacob Lebudi (Mpumalanga Province); Pieter Jooste (Northern Cape Province); Ebrahim Variava, Erna du Plessis (North West Province); Andrew Whitelaw, Kessendri Reddy, Mark Nicol, Preneshni Naicker, Colleen Bamford (Western Cape Province); Adrian Brink, Ebrahim Hoosien, Elizabeth Prentice, Inge Zietsman, Maria Botha, Peter Smith, Terry Marshall, Xoliswa Poswa (Ampath laboratories); Chetna Govind, Juanita Smit, Keshree Pillay, Sharona Seetharam, Suzy Budavari, Victoria Howell (Lancet laboratories); Carel Haumann, Catherine Samuel, Marthinus Senekal (PathCare laboratories); Andries Dreyer, Khatija Ahmed, Louis Marcus, Warren Lowman (Vermaak and Vennote laboratories); Angeliki Messina, Dena van den Bergh, and Karin Swart (Netcare hospital group); Ananta Nanoo, Andries Dreyer, Anne von Gottberg, Anthony Smith, Arvinda Sooka, Cecilia Miller, Charlotte Sriruttan, Cheryl Cohen, Chikwe Ihekweazu, Claire von Mollendorf, Desiree du Plessis, Erika van Schalkwyk, Farzana Ismail, Frans Radebe, Genevie Ntshoe, Gillian Hunt, Hlengani Mathema, Husna Ismail, Jacqueline Weyer, Jackie Kleynhans, Jenny Rossouw, John Frean, Joy Ebonwu, Judith Mwansa-Kambafwile, Karen Keddy, Kerrigan McCarthy, Liliwe Shuping, Linda de Gouveia, Linda Erasmus, Lucille Blumberg, Marshagne Smith, Martha Makgoba, Motshabi Modise, Nazir Ismail, Nelesh Govender, Neo Legare, Nicola Page, Ntsieni Ramalwa, Nuraan Paulse, Phumeza Vazi, Olga Perovic, Penny Crowther-Gibson, Portia Mutevedzi, Riyadh Manesen, Ranmini Kularatne, Ruth Mpembe, Sarona Lengana, Shabir Madhi, Shaheed Vally Omar, Sibongile Walaza, Sonwabo Lindani, Sunnieboy Njikho, Susan Meiring, Thejane Motladiile, Tiisetso Lebaka, Vanessa Quan, Verushka Chetty, Serisha Naicker, Tsidiso Maphanga, Mabatho Mhlanga, Thokozile Gloria Zulu, Ernest Tsotetsi, Phelly Matlapeng, Siphiwe Kutta, Lerato Qoza, Sydney Mogokotleng, Mbali Dube, and Amanda Shilubane (National Institute for Communicable Diseases).

This work was supported in its entirety by the National Institute for Communicable Diseases, a Division of the National Health Laboratory Service, Johannesburg, South Africa.

\section{About the Author}

Dr. Govender is a pathologist, medical epidemiologist, and head of the Centre for Healthcare-Associated Infections, Antimicrobial Resistance and Mycoses at the National Institute for Communicable Diseases, South Africa. His research interests include healthcare-associated and AIDS-related mycoses.

\section{References}

1. van Schalkwyk E, Mpembe RS, Thomas J, Shuping L, Ismail H, Lowman W, et al.; GERMS-SA. Epidemiologic shift in candidemia driven by Candida auris, South Africa, 2016-2017. Emerg Infect Dis. 2019;25:1698-707.

https:// doi.org/10.3201/eid2509.190040

2. Tsay SV, Mu Y, Williams S, Epson E, Nadle J, Bamberg WM, et al. Burden of candidemia in the United States, 2017. Clin Infect Dis. 2020;71:e449-53. https://doi.org/10.1093/cid/ ciaa193

3. Lee RA, Zurko JC, Camins BC, Griffin RL, Rodriguez JM, McCarty TP, et al. Impact of infectious disease consultation on clinical management and mortality in patients with candidemia. Clin Infect Dis. 2019;68:1585-7. https:/ / doi.org/ 10.1093/cid/ciy849

4. Kim SH, Yoon YK, Kim MJ, Sohn JW. Clinical impact of time to positivity for Candida species on mortality in patients with candidaemia. J Antimicrob Chemother. 2013;68:2890-7. https://doi.org/10.1093/jac/dkt256

5. Keighley C, Chen SCA, Marriott D, Pope A, Chapman B, Kennedy K, et al. Candidaemia and a risk predictive model for overall mortality: a prospective multicentre study. BMC Infect Dis. 2019;19:445. https:/ / doi.org/10.1186/ s12879-019-4065-5

6. Tumbarello M, Tacconelli E, de Gaetano Donati K, Morace G, Fadda G, Cauda R. Candidemia in HIV-infected subjects. Eur J Clin Microbiol Infect Dis. 1999;18:478-83. https://doi.org/10.1007/s100960050327

7. Bertagnolio S, de Gaetano Donati K, Tacconelli E, Scoppettuolo G, Posteraro B, Fadda G, et al. Hospitalacquired candidemia in HIV-infected patients. Incidence, risk factors and predictors of outcome. J Chemother. 2004;16:1728. https://doi.org/10.1179/joc.2004.16.2.172

8. Puig-Asensio M, Padilla B, Garnacho-Montero J, Zaragoza O, Aguado JM, Zaragoza R, et al.; CANDIPOP Project; GEIH-GEMICOMED (SEIMC); REIPI. Epidemiology and predictive factors for early and late mortality in Candida bloodstream infections: a population-based surveillance in Spain. Clin Microbiol Infect. 2014;20:O245-54. https://doi.org/10.1111/1469-0691.12380

9. Grim SA, Berger K, Teng C, Gupta S, Layden JE, Janda WM, et al. Timing of susceptibility-based antifungal drug administration in patients with Candida bloodstream infection: correlation with outcomes. J Antimicrob Chemother. 2012;67:707-14. https://doi.org/10.1093/jac/dkr511

10. Levine AM, Karim R, Mack W, Gravink DJ, Anastos K, Young M, et al. Neutropenia in human immunodeficiency virus infection: data from the women's interagency HIV study. Arch Intern Med. 2006;166:405-10. https://doi.org/10.1001/archinte.166.4.405

11. Kuritzkes DR. Neutropenia, neutrophil dysfunction, and bacterial infection in patients with human immunodeficiency virus disease: the role of granulocyte colony-stimulating factor. Clin Infect Dis. 2000;30:256-70. https:// doi.org/10.1086/313642 
12. Ramos A, Romero Y, Sánchez-Romero I, Fortún J, Paño JR, Pemán J, et al. Risk factors, clinical presentation and prognosis of mixed candidaemia: a population-based surveillance in Spain. Mycoses. 2016;59:636-43. https:/ / doi.org/10.1111/myc.12516

13. Meintjes G, Kerkhoff AD, Burton R, Schutz C, Boulle A, Van Wyk G, et al. HIV-related medical admissions to a South African district hospital remain frequent despite effective antiretroviral therapy scale-up. Medicine (Baltimore). 2015;94:e2269. https://doi.org/10.1097/ MD.0000000000002269

14. Ntusi N, Aubin L, Oliver S, Whitelaw A, Mendelson M. Guideline for the optimal use of blood cultures. S Afr Med J. 2010;100:839-43. https://doi.org/10.7196/SAMJ.4217

15. Vaquero-Herrero MP, Ragozzino S, Castaño-Romero F, Siller-Ruiz M, Sánchez González R, García-Sánchez JE, et al. The Pitt Bacteremia Score, Charlson Comorbidity Index and Chronic Disease Score are useful tools for the prediction of mortality in patients with Candida bloodstream infection. Mycoses. 2017;60:676-85. https://doi.org/10.1111/ myc. 12644

16. Battle SE, Augustine MR, Watson CM, Bookstaver PB, Kohn J, Owens WB, et al. Derivation of a quick Pitt bacteremia score to predict mortality in patients with Gramnegative bloodstream infection. Infection. 2019;47:571-8. https:/ / doi.org/10.1007/s15010-019-01277-7

17. Patterson L, McMullan R, Harrison DA. Individual risk factors and critical care unit effects on Invasive Candida infection occurring in critical care units in the UK: a multilevel model. Mycoses. 2019;62:790-5. https:/ / doi.org/ 10.1111/myc. 12956

18. Clinical and Laboratory Standards Institute. Performance standards for antifungal susceptibility testing of yeasts (M60). Wayne (PA): The Institute; 2017.

19. Toda M, Williams SR, Berkow EL, Farley MM, Harrison LH, Bonner L, et al. Population-based active surveillance for culture-confirmed candidemia - four sites, United States, 2012-2016. MMWR Surveill Summ. 2019;68:1-15. https://doi.org/10.15585/mmwr.ss6808a1

20. World Health Organization. Guidelines for managing advanced HIV disease and rapid initiation of antiretroviral therapy [cited 2021 Jan 14]. https:/ / www.who.int/hiv/pub/ guidelines/advanced-HIV-disease/en

21. Carmona S, Bor J, Nattey C, Maughan-Brown B, Maskew M, Fox MP, et al. Persistent high burden of advanced HIV disease among patients seeking care in South Africa's national HIV program: data from a nationwide laboratory cohort. Clin Infect Dis. 2018;66(suppl_2):S111-7.

https://doi.org/10.1093/cid/ciy045

22. Olsen MF, Abdissa A, Kæstel P, Tesfaye M, Yilma D, Girma T, et al. Effects of nutritional supplementation for HIV patients starting antiretroviral treatment: randomised controlled trial in Ethiopia. BMJ. 2014;348:g3187.

https:/ / doi.org/10.1136/bmj.g3187
23. Luzzati R, Merelli M, Ansaldi F, Rosin C, Azzini A, Cavinato $\mathrm{S}$, et al. Nosocomial candidemia in patients admitted to medicine wards compared to other wards: a multicentre study. Infection. 2016;44:747-55. https:/ / doi.org/ 10.1007/s15010-016-0924-9

24. Scudeller L, Bassetti M, Concia E, Corrao S, Cristini F, De Rosa FG, et al.; MEDICAL group; Società Italiana di Terapia Antinfettiva (SITA); Federazione delle Associazioni dei Dirigenti Ospedalieri Internisti (FADOI). MEDical wards Invasive Candidiasis ALgorithms (MEDICAL): consensus proposal for management. Eur J Intern Med. 2016;34:45-53. https:// doi.org/10.1016/j.ejim.2016.07.007

25. Sbrana F, Sozio E, Bassetti M, Ripoli A, Pieralli F, Azzini AM, et al. Independent risk factors for mortality in critically ill patients with candidemia on Italian Internal Medicine Wards. Intern Emerg Med. 2018;13:199-204. https:/ / doi.org/ 10.1007/s11739-017-1783-9

26. Naidoo K, Singh JA, Lalloo UG. Survey of ethical dilemmas facing intensivists in South Africa in the admission of patients with HIV infection requiring intensive care. South Afr J Crit Care. 2013;29:1-10. https:/ / doi.org/10.7196/ sajcc.153

27. Mkoko P, Raine RI. HIV-positive patients in the intensive care unit: a retrospective audit. S Afr Med J. 2017;107:877-81. https:/ / doi.org/10.7196/SAMJ.2017.v107i10.12298

28. Garnacho-Montero J, Díaz-Martín A, García-Cabrera E, Ruiz Pérez de Pipaón M, Hernández-Caballero C, Lepe-Jiménez JA. Impact on hospital mortality of catheter removal and adequate antifungal therapy in Candida spp. bloodstream infections. J Antimicrob Chemother. 2013;68:206-13. https:/ / doi.org/10.1093/jac/dks347

29. Lockhart SR, Etienne KA, Vallabhaneni S, Farooqi J, Chowdhary A, Govender NP, et al. Simultaneous emergence of multidrug-resistant Candida auris on 3 continents confirmed by whole-genome sequencing and epidemiological analyses. Clin Infect Dis. 2017;64:134-40. https://doi.org/10.1093/cid/ciw691

30. Govender NP, Magobo RE, Mpembe R, Mhlanga M, Matlapeng P, Corcoran C, et al. Candida auris in South Africa, 2012-2016. Emerg Infect Dis. 2018;24:2036-40. https://doi.org/10.3201/eid2411.180368

31. Govender NP, Patel J, Magobo RE, Naicker S, Wadula J, Whitelaw A, et al.; TRAC-South Africa group. Emergence of azole-resistant Candida parapsilosis causing bloodstream infection: results from laboratory-based sentinel surveillance in South Africa. J Antimicrob Chemother. 2016;71:1994-2004. https://doi.org/10.1093/jac/dkw091

Address for correspondence: Nelesh P. Govender, Centre for Healthcare-Associated Infections, Antimicrobial Resistance and Mycoses, National Institute for Communicable Diseases, Private Bag X4, Sandringham, 2132, South Africa; email: neleshg@nicd.ac.za 